\title{
Rare Location of Castleman's Disease in the Temporal Region: A Case Report Involving a Young Korean Woman and Review of the Literature
}

Wan Cheol Ryu ${ }^{1}$,

Moon Hyang Park ${ }^{2^{*}}$,

Hoon $\mathrm{Kim}^{1}$,

In Chang Koh ${ }^{1}$,

Kyu Nam Kim ${ }^{1^{*}}$

Departments of

${ }^{1}$ Plastic and Reconstructive Surgery and

${ }^{2}$ Pathology, Konyang University Hospital,

Konyang University of College of Medicine,

Daejeon, Korea

No potential conflict of interest relevant to this article was reported.
Castleman's disease (CD) is an uncommon benign lymphoproliferative disorder of unknown etiology. Histopathologically, it is divided into three types: hyaline-vascular, plasma cellular, and multicentric $C D$. The mass usually presents asymptomatically; however, it can cause non-specific symptoms such as fever and fatigue. Although CD can be found wherever lymph nodes are present, $75 \%$ of cases are reported in the mediastinum, and occurrence in the head and neck is rare. Herein, we report a rare case of CD presenting as a superficial mass in the temporal region. To the best of our knowledge, this is the first report of temporal $\mathrm{CD}$ in Korea involving a young patient.

Keywords: Giant lymph node hyperplasia / Temporal region

\section{INTRODUCTION}

Castleman's disease (CD), also known as "giant lymph node hyperplasia", "angiomatous lymphoid hamartoma", and "angiofollicular lymph node hyperplasia" is a rare benign lymphoproliferative disorder of unknown etiology. It was first described in 1954 as a form of benign lymph node hyperplasia simulating thymoma [1]. Approximately $75 \%$ of all cases are found in the mediastinum; $15 \%$ are found in the abdomen, retroperitoneum, or pelvis; and $10 \%$ occur in the head and neck. A few isolated cases have also been described involving extra-lymphatic tissues, including the lung, larynx, parotid gland, pancreas, and muscle [2]. Herein, we

\footnotetext{
Correspondence: Kyu Nam Kim

Department of Plastic and Reconstructive Surgery, Konyang Hospital, Konyang University of College of Medicine, 158 Gwanjeo Dong-ro, Seo-gu, Daejeon 35365, Korea E-mail:manabear77@naver.com

*Moon Hyang Park and Kyu Nam Kim contributed equally to this work.

Received January 10, 2017 / Revised April 13, 2017 / Accepted April 16, 2017
}

present a rare case of $\mathrm{CD}$ presenting as a superficial mass in the temporal region. To the best of our knowledge, this is the first report of temporal $\mathrm{CD}$ in Korea involving a young patient.

\section{CASE REPORT}

A 42-year-old Korean woman presented to our department with a slow-growing mass on her left temporal region over a duration of 1 year. The mass was approximately $2.0 \times 1.5 \mathrm{~cm}$ in size, protruding, soft, and non-tender. She had no systemic symptoms such as headache, weight loss, fatigue, or fever. Contrast-enhanced computed tomography (CT) of the head and neck revealed a superficial and well-enhanced homogenous mass (Fig. 1). This lesion may be radiologically discriminated as a schwannoma, hemangioma, reactive lymph node, or lymphoma.

We performed an excisional biopsy based on the clinical impression of a benign superficial soft tissue mass. Following exci- 
sion, a $2.1 \times 1.5 \mathrm{~cm}$-sized cavity formed. To obliterate the expected dead space, we performed a marginal de-epithelialized buried flap. The wound was closed primarily with subcutaneous and skin sutures without tension. A silastic drain was inserted to pre- vent hematoma; it was removed one day after the surgery (Fig. 2). No postoperative complications such as hematoma, seroma, infection, or dehiscence was reported.

Histopathological examination revealed a lymph node with
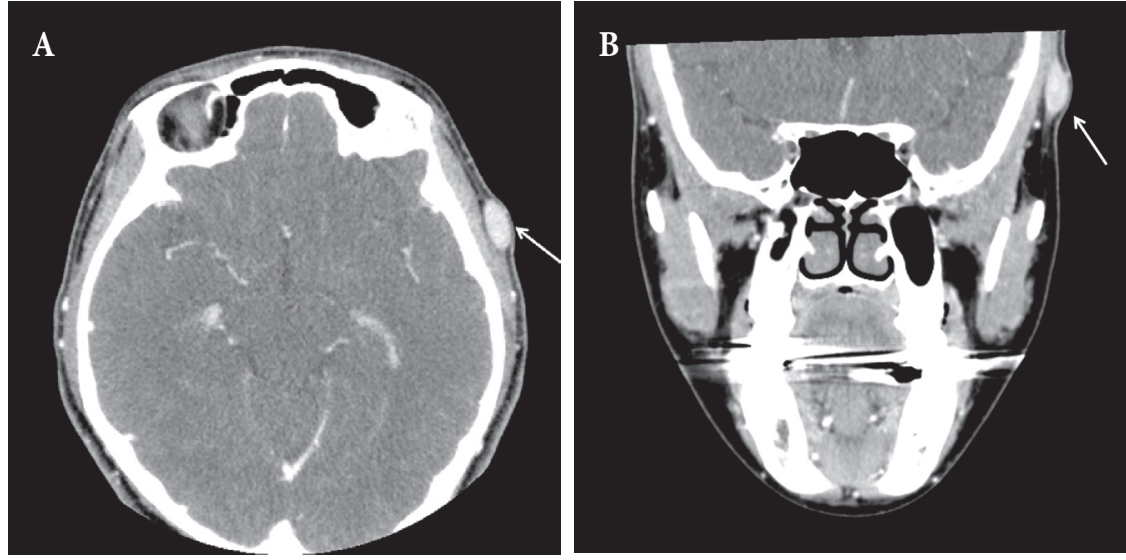

Fig. 1. Preoperative computed tomography image showing an approximately $1.8 \times 0.8 \mathrm{~cm}$ in size, well-demarcated mass involving the left temporal region. A well-enhanced homogenous mass in the subcutaneous area (white arrows). (A) Axial view. (B) Coronal view.
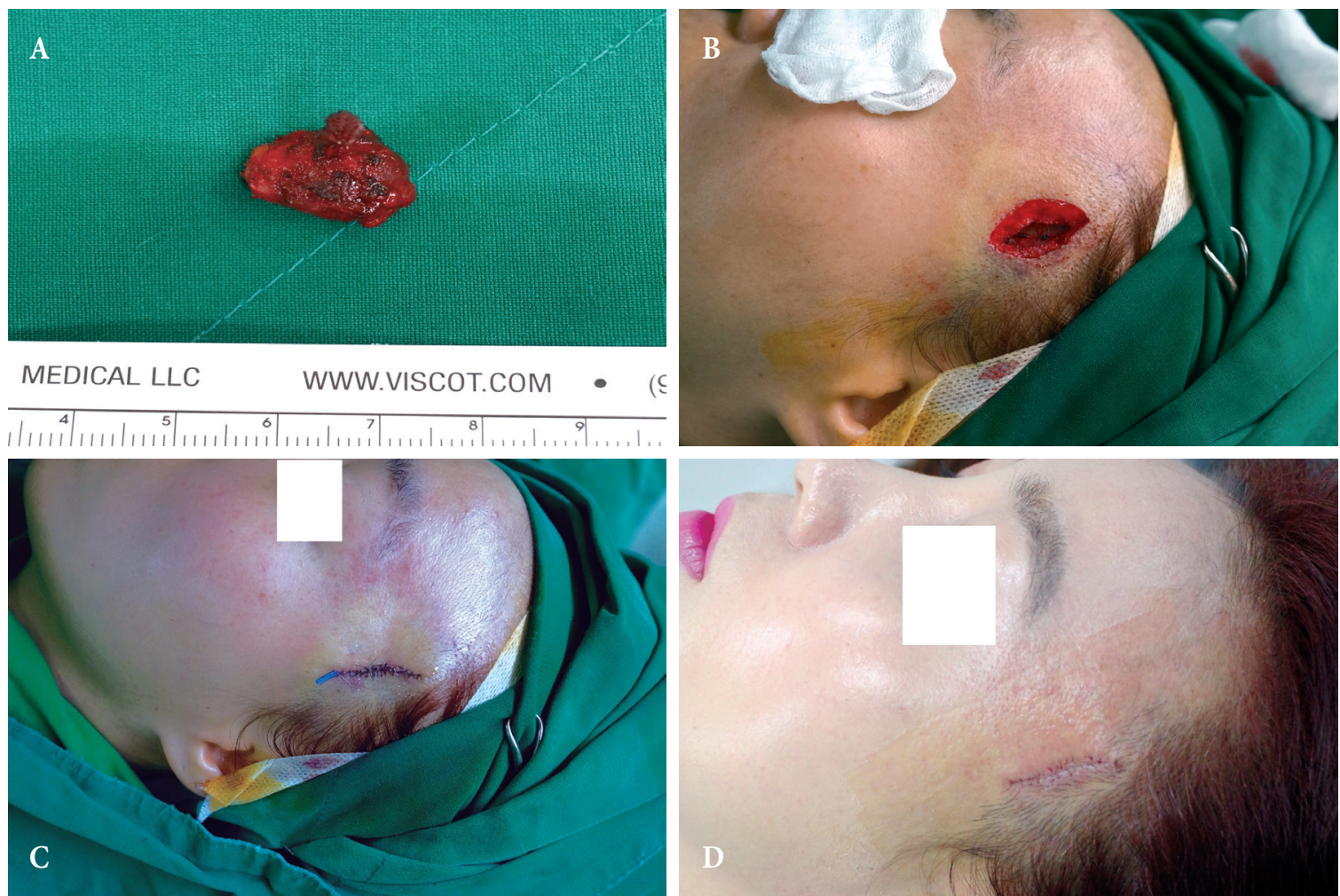

Fig. 2. Clinical photographs showing. (A) A well-circumscribed, firm, ovoid mass measuring $19 \times 8 \times 7 \mathrm{~mm}$ in dimensions. (B) The marginal de-epithelialized buried flap, which was performed to obliterate the expected dead space. (C) Tension-free primary closure with a silastic drain inserted. (D) Postoperative photograph at 3 weeks follow-up. 

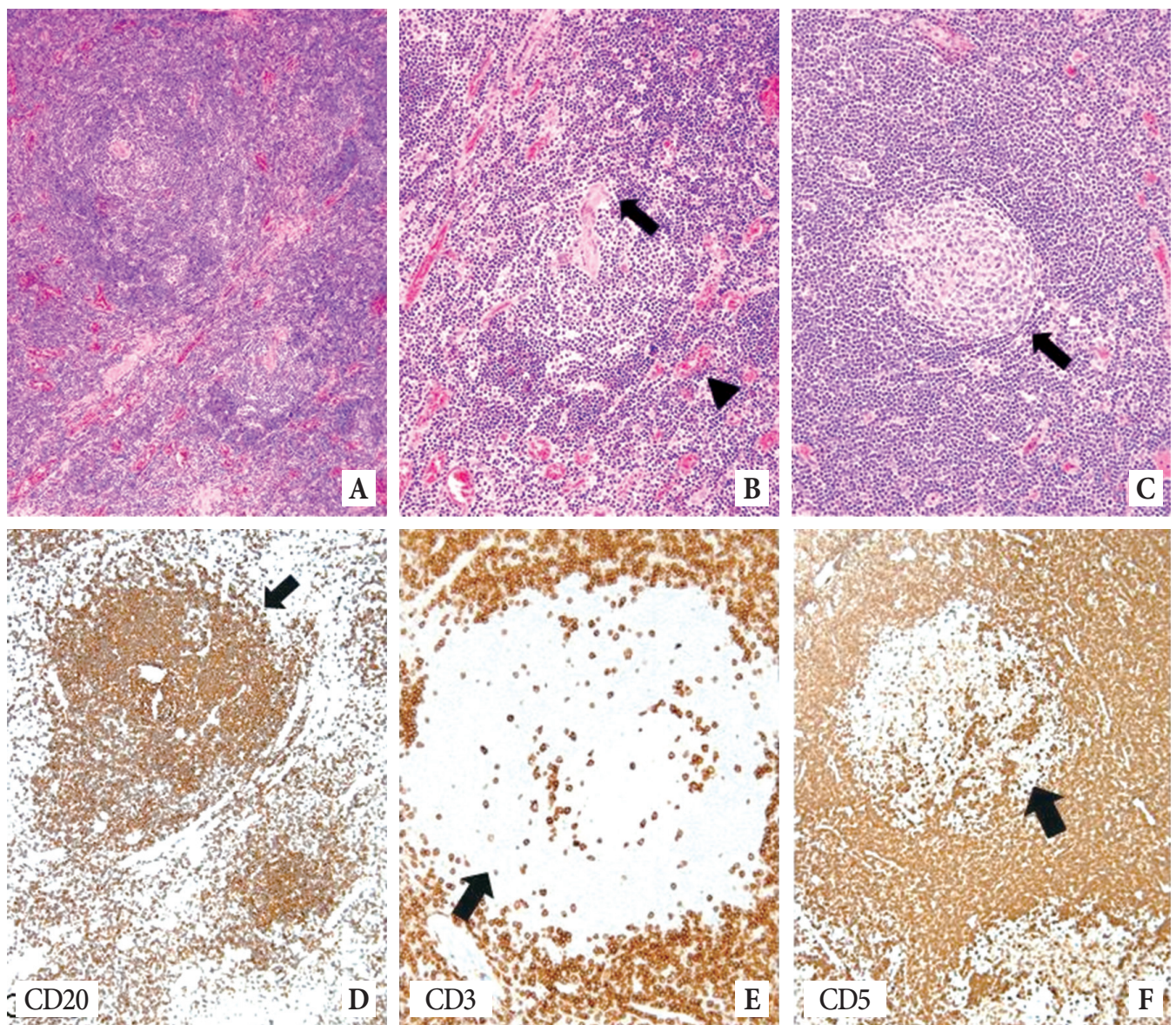

Fig. 3. Microscopic features of Castleman's disease of the hyaline-vascular type. (A) The interfollicular areas are composed of a network of vessels $(\mathrm{H} \& \mathrm{E}, \times 100)$. (B) Small regressed germinal centers with penetrating vessels (arrow with a tail) and onion-skin layering of the mantle cells (arrow without a tail) $(\mathrm{H} \& \mathrm{E}, \times 200)$. (C) The germinal centers appear to be atrophic (arrow) (H\&E, $\times 200)$. (D) The follicles are positive for CD20 (arrow) (immunohistochemical staining, $\times 100$ ). (E) The mantle zone is negative for CD3 (arrow) (immunohistochemical staining, $\times 200$ ). (F) Positivity for CD5 (arrow) (immunohistochemical staining $\times 100$ ).

relatively well-preserved lymphoid architecture, showing follicles of varying sizes with atrophic and often vascularized germinal centers. The germinal centers appeared to be atrophic, with penetrating blood vessels and surrounded by a cup of small lymphocytes that were arranged in a concentric "onion skin" layer. The follicular tissues were composed of a network of vessels on a thick collagenous scaffold; sinus structures were not identified. Immunohistochemistry revealed the follicles to be positive for CD20. The mantle zone was negative for $\mathrm{CD} 3$ but positive for $\mathrm{CD} 5$. These histopathological findings corresponded with hyaline-vascular CD, which was confirmed by our senior pathologist (M.H.P.) (Fig. 3). The patient experienced no lesion recurrence at 6 months' follow-up, and reported satisfaction with the appearance of her surgical site contour and scar.

\section{DISCUSSION}

In 1956, Castleman et al. [1] first reported a case of "localized mediastinal lymph node hyperplasia resembling thymoma". These lesions occur within the mediastinum, neck, axilla, pelvis, and retroperitoneum [3]. According to recent literature, the incidence rate of $\mathrm{CD}$ is approximately 21 persons per one million. Localized $\mathrm{CD}$ occurs in 15.9 persons per one million, while multicentric $\mathrm{CD}$ occurs in 5.1 persons per one million [4].

$\mathrm{CD}$ is divided into 3 subtypes: hyaline-vascular CD (HVCD), plasma cell CD (PCCD), and multicentric CD. The pathogenesis 
of CD is still unclear. One theory suggests that it is a result of chronic antigenic stimulation, like virus antigen, while other theories focus on nodal developmental growth disturbances. Excessive production of interleukin 6 (IL-6) by lymph node hyperplasia has been implicated in the pathogenesis of this disease. Additionally, CD has relevant human herpes virus 8 (HHV-8) or Kaposi sarcoma-associated virus, especially the multicentric type [5]. Interestingly, recent evidence indicates that excessive release of IL-6 may not only be evident in CD, but may actually underlie the pathophysiology of both localized and multicentric CDs. In earlier studies, patients with systemic symptoms and localized CD were shown to have a reduction in symptoms associated with decreased IL-6 levels following excision of the abnormal node $[4,6]$.

Localized CD is often diagnosed incidentally via radiographical examination due to its asymptomatic feature. The most common histopathological type is HVCD, while 10\%-20\% are PCCD. The latter appears to be more closely-linked with systemic signs and symptoms such as fever, arthralgia, fatigue, anemia, thrombocytosis, and hypergammaglobulinemia [7]. The treatment of choice for localized CD is surgical excision, with a combination of radiotherapy, as required. Surgery is curative when resection is complete, yielding a 5-year survival rate close to $100 \%$, with recurrences being infrequent. Patients without systemic involvement should have an additional radiological assessment six to twelve months after the initial therapy, to verify that there is no recurrence. Localized CD's prognosis is good [8].

Multicentric CD usually occurs as a systemic lesion. The clinical manifestations are very diverse; some rapidly-progress to death within a few weeks, while others grow relatively slowly over several years. Therefore, it is clinically important to discriminate between localized $\mathrm{CD}$ and multicentric $\mathrm{CD}$, given the distinction in both their treatment and prognoses. Localized CD has a generally good prognosis as it can be treated with surgery and radiotherapy, with a reported 5-year overall survival of 91\% [9]. Multicentric CD has a relatively worse prognosis than localized CD; its primary treatment consists of a combination of immunotherapy and chemotherapy. Recently, treatment combinations of chemotherapy with therapy targeted to CD20 (rituximab) or IL-6 receptor (tocilizumab) have been attempted [10]. The 5-year survival rate of patients with multicentric CD was reported to be $65 \%$. Patients who present with negative prognostic factors (e.g., HIV, HHV-8, or Kaposi sarcoma-associated herpes virus) have a reported median survival of approximately $20 \%$ [9].

Furthermore, although CD is considered a benign disease of the lymph nodes, it could be associated with other disease processes. According to one literature, eight patients with localized $\mathrm{CD}$ were shown to have associated malignant lymphoma, six with B-cell non-Hodgkin lymphoma, and two with Hodgkin lymphoma. In addition, a minority of localized CD patients (approximately 15\%) developed paraneoplastic pemphigus [11].

We conducted a literature review using PubMed and KoreaMed from their inception to December 31st, 2016. The search terms included "giant lymph node hyperplasia" OR "Castleman disease" AND "face" OR "neck" AND "Korea”. A total of 35 articles and 56 cases of head and neck CD were reported in Korea. Of these, 46, 4, and 3 cases involved the neck, orbit, and parotid areas, respectively, while the nasopharynx, mandible, and temporal areas were represented by 1 case each. According to our literature review, Kang et. al. [12] was the first to report a case of CD occurring in the temporal region, in a 69-year-old man. Compared to their report, our patient was a relatively young woman. In addition, we shared the gross macroscopic appearance of the specimen (Fig. 2A), and we also closed the wound with a marginal de-epithelialized buried flap, in order to obliterate the expected dead space, thus achieving a satisfactory aesthetic outcome. As mentioned earlier, while there have been several reports of CD involving the neck area in Korea, only one case of CD involving the temporal region has been reported previously. We thus believe that our report is significant in terms of CD occurring in an uncommon anatomical area, whereby the patient presented with a superficial mass in the temporal region (Table 1).

As demonstrated in our case, the diagnosis of CD may be difficult through initial clinical evaluation and imaging work-up. Therefore, plastic surgeons and pathologists should be aware of $\mathrm{CD}$ when presented with an asymptomatic mass. It is necessary to confirm CD through excisional biopsy and histopathological examination. Additionally, proper medical work-up and follow-up of the patient should be conducted after excision. 
Table 1. Review of head and neck CD in Korea

\begin{tabular}{|c|c|c|c|c|c|c|}
\hline Study & Year & Journal & Study design & Histological type & Site & No. of cases \\
\hline Son HW et. al. & 2015 & KJA & Case report & PCCD & Neck & 1 \\
\hline Lee SK et. al. & 2013 & JKSR & Case report & HVCD & Neck & 1 \\
\hline O Kyu Noh et. al. & 2013 & ROJ & Case report & HVCD & Neck & 1 \\
\hline Park SH et. al. & 2013 & $J R D$ & Case report & HVCD & Neck & 1 \\
\hline Yun SH et. al. & 2012 & JKORL & Case report & HVCD & Neck & 1 \\
\hline Kim U et. al. & 2010 & KJO & Case report & PCCD & Neck & 1 \\
\hline Park SH et. al. & 2010 & KJO & Case report & PCCD & Orbit & 1 \\
\hline Ro HW et. al. & 2010 & JKSS & Case report & HVCD & Neck & 1 \\
\hline Kim B et. al. & 2009 & JKMS & Case report & PCCD & Neck & 1 \\
\hline Cha DM et. al. & 2009 & JKOS & Case report & HVCD & Orbit & 2 \\
\hline Kang NH et. al. & 2009 & JCFS & Case report & HVCD & Temporal area & 1 \\
\hline Kim SH et. al. & 2009 & JKORL & Case report & HVCD & Nasopharynx & 1 \\
\hline Park JH et. al. & 2008 & ANL & Case report & HVCD & Pariotid & 1 \\
\hline Yang JH et. al. & 2008 & $\mathrm{KJH}$ & Case report & PCCD & Neck & 1 \\
\hline Jung JH et. al. & 2007 & K J Pathol. & Case report & HVCD & Neck & 1 \\
\hline Song JJ et. al. & 2006 & EAO & Case series & HVCD & Neck and parotid & 12 \\
\hline Kim JY et. al. & 2005 & JKORL & Case series & HVCD & Neck & 6 \\
\hline Koo SE et. al. & 2005 & KJP & Case report & HVCD, PCCD & Neck & 1 \\
\hline Son EJ et. al. & 2004 & JKORL & Case report & HVCD & Neck & 1 \\
\hline Park SY et. al. & 2004 & JKORL. & Case report & HVCD & Neck & 1 \\
\hline Chae BJ et. al. & 2004 & JKORL & Case report & HVCD & Neck & 1 \\
\hline Kim EA et. al. & 2003 & KJP & Case report & HVCD & Neck & 1 \\
\hline Park KS et. al. & 2002 & AOS & Case report & HVCD & Orbit & 1 \\
\hline Chung IK et. al. & 2001 & JKAOMS & Case report & HVCD, PCCD & Mandible, orbit & 1 \\
\hline Kim JE et. al. & 2000 & JKMS & Retrospective cohort & PCCD & Neck & 8 \\
\hline Kwon SJ et. al. & 1999 & JKMS & Case report & PCCD & Neck & 1 \\
\hline Choi G et. al. & 1997 & $\mathrm{JOL}$ & Case report & HVCD & Parotid & 1 \\
\hline Kim HJ et. al. & 1997 & JKSR & Case report & HVCD & Neck & 1 \\
\hline Sohn WY et. al. & 1997 & JKSR & Case report & PCCD & Neck & 1 \\
\hline Oh SS et. al. & 1993 & JKSR & Case report & PCCD & Neck & 1 \\
\hline Kim MS et. al. & 1992 & JKORL & Case report & HVCD & Neck & 1 \\
\hline Lee JW et. al. & 1992 & JKORL & Case report & PCCD & Neck & 1 \\
\hline Cho JH et. al. & 1990 & K J Pathol. & Case series & HVCD & Neck & 5 \\
\hline An HK et. al. & 1989 & K J Pathol. & Case series & HVCD & Neck & 1 \\
\hline Gil HJ et. al. & 1987 & JKSR & Case report & PCCD & Neck & 1 \\
\hline Total & & & & & & 56 \\
\hline
\end{tabular}

CD, Castleman's disease; KJA, Korean Journal of Anesthesiology; PCCD, plasma cell CD; JKSR, Journal of Korean Society of Radiology; HVCD, hyaline-vascular CD; ROJ, Radiation Oncology Journal; JRD, Journal of Rheumatic Diseases; JKORL, Korean Journal of Otorhinolaryngology-Head and Neck Surgery; KJO, Korean Journal of Ophthalmology; JKSS, Journal of the Korean Surgical Society; JKMS, Journal of Korean Medical Science; JKOS, Journal of Korean Ophthalmological Society; JCFS, Journal of Craniofacial Surgery; ANL, Auris Nasus Larynx; KJH, Korean Journal of Hematology; K J Pathol., Korean Journal of Pathology; EAO, European Archives Oto-rhino-laryngology; KJP, Korean Journal of Pediatrics; AOS, Acta Ophthalmologica Scandinavica; JKAOMS, Journal of Korean Association of Oral and Maxillofacial Surgeons; JOL, Journal of Otolaryngology; JKSR, Journal of Korean Society of Radiology. 


\section{REFERENCES}

1. Castleman B, Iverson L, Menendez VP. Localized mediastinal lymphnode hyperplasia resembling thymoma. Cancer 1956;9:822-30.

2. McAdams HP, Rosado-de-Christenson M, Fishback NF, Templeton PA. Castleman disease of the thorax: radiologic features with clinical and histopathologic correlation. Radiology 1998;209:221-8.

3. Keller AR, Hochholzer L, Castleman B. Hyaline-vascular and plasmacell types of giant lymph node hyperplasia of the mediastinum and other locations. Cancer 1972;29:670-83.

4. Munshi N, Mehra M, van de Velde H, Desai A, Potluri R, Vermeulen J. Use of a claims database to characterize and estimate the incidence rate for Castleman disease. Leuk Lymphoma 2015;56:1252-60.

5. Song JJ, Jung MH, Woo JS, Chae SW, Hwang SJ, Lee HM. Castleman's disease of the head and neck. Eur Arch Otorhinolaryngol 2006;263: 160-3.

6. Yoshizaki K, Matsuda T, Nishimoto N, Kuritani T, Taeho L, Aozasa K, et al. Pathogenic significance of interleukin-6 (IL-6/BSF-2) in Castleman's disease. Blood 1989;74:1360-7.
7. Puram SV, Hasserjian RP, Faquin WC, Lin HW, Rocco JW. Castleman disease presenting in the neck: report of a case and review of the literature. Am J Otolaryngol 2013;34:239-44.

8. Bowne WB, Lewis JJ, Filippa DA, Niesvizky R, Brooks AD, Burt ME, et al. The management of unicentric and multicentric Castleman's disease: a report of 16 cases and a review of the literature. Cancer 1999;85:706-17.

9. Shin DY, Jeon YK, Hong YS, Kim TM, Lee SH, Kim DW, et al. Clinical dissection of multicentric Castleman disease. Leuk Lymphoma 2011;52:1517-22.

10. Chen YF, Zhang WD, Sun CZ, Ouyang D, Chen WK, Luo RZ, et al. Clinical features and outcomes of head and neck castleman disease. J Oral Maxillofac Surg 2012;70:2466-79.

11. Larroche C, Cacoub P, Soulier J, Oksenhendler E, Clauvel JP, Piette JC, et al. Castleman's disease and lymphoma: report of eight cases in HIVnegative patients and literature review. Am J Hematol 2002;69:119-26.

12. Kang N, Chung JW, Jung SN. Rare location of Castleman disease in the temporal region. JCraniofac Surg 2009;20:830-2. 